\title{
In vivo assessment of thickness of the psoas major muscle in adult male subjects: evaluating occupation-based activity-related differences
}

\author{
Ogugua Augustine Egwu, Albert Nkereuwem Eteudo, Gabriel Godson Akunna, Obinna Uchewa
}

Department of Anatomy, Federal University, Ndufu-Alike Ikwo, Abakaliki, Nigeria

Submitted: 4 December 2016

Accepted: 23 February 2017

Arch Med Sci Civil Dis 2017; 2: e41-e47

DOI: https://doi.org/10.5114/amscd.2017.66551

Copyright @ 2017 Termedia \& Banach

\section{Abstract}

Introduction: There is a notable scarcity of in vivo studies on the muscles of the posterior abdominal wall, and the psoas major muscle, which is important in occupation-related lumbopelvic mechanics, is not an exception. Hence, we determined the psoas major muscle thickness (PMMT) in an adult Nigerian population of different occupational groups with the aim of assessing the effect of unregulated occupation-related physical activity on the muscle while identifying its relationships with known anthropometric variables.

Material and methods: Eighty apparently healthy males between the ages of 18 and 30 were recruited for the study and divided into two groups: a control group of 40 relatively inactive subjects who were students and an age-matched quasi-experimental group of 40 artisans (manual labourers). B-mode ultrasound was used to measure the thickness of the psoas major muscle at the point of maximum thickness.

Results: The mean PMMT for the control group was $31.74 \pm 7.91 \mathrm{~mm}$ and did not significantly correlate with body mass index $(r=-0.151, p<0.05)$, body surface area $(r=-0.255, p>0.05)$, height $(r=-0.186, p>0.05)$, weight $(r=-0.244, p>0.05)$, or age $(r=0.159, p>0.05)$. However, PMMT was significantly greater in the artisan group $(40.10 \pm 4.89 \mathrm{~mm})$ than in the control group (31.74 $\pm 7.91 \mathrm{~mm})$ at $p<0.05$.

Conclusions: This result is an obvious indication of the effect of unregulated occupation-related physical activity on the muscle, which may lead to a gradual alteration of normal lumbopelvic mechanics in these individuals.

Key words: psoas major, psoas major thickness, ultrasound, in vivo, Nigeria.

\section{Introduction}

Some researchers and anatomists still refer to the hip flexor muscle complex as one unit or as the iliopsoas [1, 2]. The psoas muscle differs from the iliacus in that it has a different architecture, innervations and more importantly a different function. The psoas muscle comprises both the psoas major and minor, but as the psoas minor is absent in about $40 \%$ of individuals [3], this paper will focus on the psoas major muscle. A better understanding of the roles of the psoas major muscle and its impact on lumbopelvic stability may improve the clinical management of individuals suffering from lower back pain. Muscle tissues are the major attachments to the framework of the human body, also known as the

\author{
Corresponding author: \\ Ogugua Augustine Egwu \\ Department of Anatomy \\ Federal University \\ Ndufu-Alike Ikwo \\ P.M.B 1010 \\ 234 Abakaliki, Nigeria \\ Phone: +2348034518414 \\ E-mail: egwuoc@gmail.com
}


bony skeleton, and form the structures of visceral components of the body. Muscle tissues are made up of contractile cells which also produce movement or force to propel the body. The majority of muscles are attached to the skeletal system of the body, with a few forming the architecture of some visceral organs. Therefore muscle can be classified into three major classes: the skeletal muscles, smooth muscle and circular or cardiac muscles. While the skeletal muscle is attached to the bony skeleton, the smooth muscles form the visceral component and the cardiac muscle is present in the heart.

Skeletal muscle, which is also known as striated muscle, dominates the functional role of muscles in the body as a source of movement. Skeletal muscle architecture is the primary determinant of muscle function [4]. Morphological characteristics of muscles are usually fascicle length, pennation angle and the thickness of the muscle $[3,5]$.

Unfortunately, the knowledge of human muscle architecture has until recently been based on the dissection of cadaver specimens or biopsies [5]. The knowledge of muscle architecture through cadaver dissection use is grossly unreliable due to the shrinkage of embalmed cadaver muscle tissues [5]. Therefore, it is necessary to obtain a more accurate and reliable understanding of muscle architecture including its thickness, length, and volume in living human bodies through ultrasound.

The psoas major muscle is one of the muscular components of the posterior abdominal wall (the psoas major, psoas minor, the iliacus and the quadratus lumborum). The psoas major lies in the posterior abdominal wall and is related anteriorly to the diaphragm and pleura. The posterior abdominal wall is muscular and supports not only the retroperitoneal organs such as the kidney, ureter and duodenum but also all the other organs and vessels of the biggest cavity of the body [3].

The psoas major is a fusiform muscle located on the side of the lumbar spine and along the brim of the pelvis. The psoas and the iliacus are together known as the iliopsoas due to their common insertion and actions. The psoas major muscle passes behind the inguinal ligament and in front of the hip joint to enter the thigh. It ends in a tendon which receives the fibres of the iliacus on its central side, therefore forming the iliopsoas muscle. The iliopsoas tendon then inserts into the tip and medial part of the anterior surface of the lesser trochanter of the femur [4].

The iliopsoas acts as a powerful flexor of the hip joint as in raising the trunk from a leaning to a sitting posture [5]. The psoas is a major contributor to maintaining the stability at the hip. It balances the trunk while sitting. The psoas can also bring about the lateral flexion of the trunk when muscle of one side is acting in isolation of the other. It is a weak medial rotator of the hip. After fracture of the neck of the femur the limb rotates laterally [4].

Furthermore, neither cadaver muscles nor biopsy specimens allow muscle fibre morphology and force development to be studied during contraction in the living human being [6]. In vivo muscle and cadaver muscle architectural characteristics differ in both contracted and relaxed conditions [7]. Therefore, the non-invasive method on living subjects is required to study muscle changes in architecture during force development.

Ultrasonography has been in medical practice since the early 1950s when Wild and Neal discovered the ability of high-frequency ultrasound waves to visualize living tissues [8]. Since then, the technique of ultrasound has rapidly developed, leading to its widespread use in almost all fields of medicine because of its non-invasive nature and real time display. Diseased muscle showed a different ultrasound appearance when compared to that of healthy muscle [9]. Ultrasound can now detect neuromuscular disorders, malignancies, infections, hematomas and ruptures of the musculoskeletal system [10-12]. The sonographic appearance of muscles is fairly distinct and can easily be discriminated from surrounding structures such as subcutaneous fat, bone, nerves and blood vessels [12]. Normal muscle is relatively black and has low echo intensity [13].

Ultrasound is a quick, relatively inexpensive, safe (non-radiation danger), non-invasive and widely accessible imaging technique for muscle assessment [2]. The high acoustic contrast of muscle with the adjoining tissue makes it particularly suitable for ultrasonographic evaluation [2]. Ultrasound imaging has greater spatial resolution for superficial muscle structures than magnetic resonance imaging (MRI) and also allows for dynamic normal examination with real-life feedback [2]. The knowledge of muscle morphology including its dynamism in force generation and possible role in environmental/occupational and sports medicine is vital.

Using ultrasonography to access the thickness of the psoas major muscle will elucidate the understanding of the normal thickness of the psoas major muscle, and also identify different variations in the thickness and size of the psoas major muscle. Studies have established the reliability of ultrasound as a good technique for measuring psoas major muscle thickness; its benefit of easy reproducibility by practitioners of varying experience, especially in areas such as radiographic anatomy, musculoskeletal therapy and sports physiotherapy, has been established [14]. Furthermore, the effect of physical activity on the muscle thickness could be determined, as previous stud- 
ies have reported that occupation could affect the structural disposition of muscles [15].

This study was therefore conducted to investigate the thickness of the psoas major muscle among young healthy adult Nigerians using ultrasound. The thickness of the muscles will possibly be a pointer to the functional status of the muscle, its use among young adults, its possible relationship with iliopsoas tendon thickness and its role in lower back pain. To the best of our knowledge, no measurement relating to the thickness of the psoas major muscle has been established and no ultrasound-based evaluation of the thickness of the psoas major muscle among young adult Nigerians has been reported in the literature - hence the need to carry out this study.

\section{Material and methods}

\section{Materials}

A brightness mode (B-mode) ultrasound machine (Siemens Sonoline) was used to measure the thickness of psoas major muscles.

\section{Study area}

This study was carried out in Abakaliki, the capital city of Ebonyi State, which is a mainland south-eastern state of Nigeria mainly inhabited by the Igbos of southern Nigeria. It lies approximately within longitudes $7^{\circ} 30^{\prime}$ and $7^{\circ} 30^{\prime} \mathrm{E}$ and latitudes $5^{\circ} 40^{\prime}$ and $6^{\circ} 45^{\prime} \mathrm{N}$. It has a landmass of about 5,935 square kilometres. The population of Ebonyi State according to the National Population Commission held on $21^{\text {st }}$ March, 2006 is about 2,176,947 people with males numbering 1,064,156 and females $1,112,791$. Agriculture is the major economic base of the state with about $80 \%$ of the population actively engaged. It is a leading producer of rice, yams, potatoes, maize, beans, cassava, etc, while those inhabiting the riverine area are actively engaged in fish farming. Ebonyi State is boarded to the east by Cross-river State, to the west by Enugu State, to the north by Benue State and to the south by Imo State and Abia State.

The study centre was at Life Scan Ultrasound Centre, Felix Memorial Hospital Hilltop Road, Abakaliki, Ebonyi State, Southeast, Nigeria. It serves as the imaging unit of the Department of Anatomy, Ebonyi State University, Abakaliki. This centre receives patients from within Abakaliki metropolis and beyond. They are mostly obstetric patients and individuals with soft tissue pathology from private hospitals within the town and from other cities outside Abakaliki Capital territory.

\section{Subjects}

The sampling method is that of a convenient purposive nature. Eighty healthy subjects were se- lected from a pool of students in College of Health Science, PRESCO Campus, Ebonyi State University, Abakaliki, Southeast, Nigeria and were recruited for this study. All subjects were physically active, not previously involved in regular weight training (weight lifting). None had any subjective clinical evidence of musculoskeletal injury or any orthopaedic abnormality. They were advised to avoid engaging in arduous physical activity or exercises such as sit-ups, lifting heavy weight objects, pulling or pushing heavy objects, etc, 2 weeks before the study, as this is found to cause an increase in muscle thickness [16].

\section{Instrumentation}

A B-mode ultrasound system (Siemens Sonoline SL-I version 2000) was used to measure psoas major muscle thickness with a $7.5 \mathrm{MHz}$ linear transducer.

\section{Scanning protocol}

To ensure reproducibility of results and clinical relevance we adhered to the National Centre for Health Statistics and WHO standards. The subjects lay in a supine position. This position is chosen to facilitate contact between the probe and the muscle to avoid an anisotropic effect, which can occur if the muscle is shortened in a sitting position.

The ultrasound gel was applied on the surface and the transducer. A transverse view of the psoas major was viewed with the transducer placed in the transverse plane. The measurements were taken at the point where the psoas major muscle has its thickest measurement at the level of L4-L5 of the lumbar vertebrae. The thickest point of the psoas major is between lumbar vertebrae 4 and 5 ( $\mathrm{L} 4$ and L5) taken from anterior surface landmark of 5-7 cm superolateral to the umbilicus (at an angle between $25^{\circ}$ and $30^{\circ}$ ) at the midclavicular line. All measurements were taken by one person to avoid inter-observer variability.

Probe position - midclavicular line, superolateral to the umbilicus at an angle ranging between $25^{\circ}$ and $30^{\circ}$ with a distance of $5-7 \mathrm{~cm}$.

\section{Sketched diagram of psoas major muscle surface landmark}

The surface landmark of the psoas major muscle was taken at the midclavicular line superolateral to the umbilicus at a horizontal distance of about $5-7 \mathrm{~cm}$ and at an angle of between $25^{\circ}$ and $30^{\circ}$ (Figure 1).

\section{Anthropometric measurement}

Anthropometric measurements of some parameters such as age, height and weight were all 


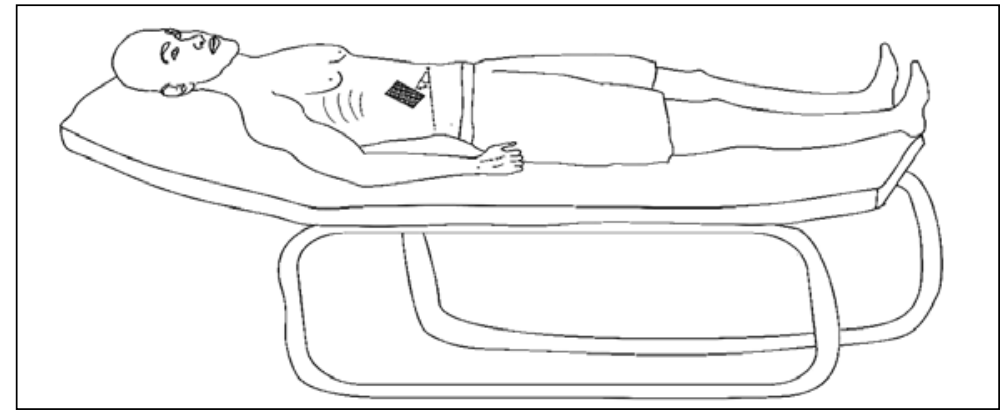

Figure 1. Anatomical landmark where measurement was taken

measured on the right side of the body of the subjects for data analysis.

\section{Weight}

The body weight was measured using a body weight scale. This scale was at the zero scale reading before measuring each subject. The subjects were asked to remove any heavy material from their pocket (keys, wallets, chain belts, phones, etc) and heavy clothing or apparel such as a large jacket, shoes, woollen jersey, etc, as that could alter the reading accuracy. The subjects were all measured in the morning. They were asked to stand erect, and look at eye level (straightforward) while standing on the scale. The readings were taken when the pointer/digital screen settled.

\section{Height}

Height was measured using a measuring tape. This was taken by carefully calibrating the wall with the tape from ground level to a height of $3 \mathrm{~m}$. The calibrated wall was then used as a focal point for measuring the subjects' height. Prior to the measurement the subjects were asked to remove their shoes and caps to avoid error in reading. While taking the measurement they were asked to stand with their back to the wall; the back of their feet, calves, buttocks, upper back and back of their heel were all in contact with the wall. A meter rule was used as a pointer to rest on top of the head and point at the wall while the reading was then taken.

Anthropometric measurements were taken twice and the average deduced and reported in line with international best practices.

\section{Ethical approval}

In line with the Helsinki Declaration of 2014 where respect for persons, beneficence and justice are recommended in all research involving human subjects, ethical approval was obtained from the Ethical/Research Committee of the Faculty of Basic Medical Science, Ebonyi State University, Abakaliki, Southeast, Nigeria.

\section{Statistical analysis}

All the data collected for each subject were compiled in a Microsoft Excel spreadsheet. For statistical analysis, the software Statistical Package for the Social Sciences (SPSS) version 20 was used. The mean, standard deviation and correlations were calculated. The result was presented as mean \pm standard deviation and correlations were calculated using the bivariate Pearson's method for correlations at both significant levels 0.05 and 0.01 (or 1 tailed).

\section{Results}

A total of 80 young male adult subjects of south-eastern Nigeria between the ages of 18 and 35 years were recruited for this study. Forty people in the control group and 40 people who engaged in hard labour in the artisan group constituted the quasi-experimental group. The thickness of the psoas major muscle $(\mathrm{mm})$ in the controls was reported; the relationship between the anthropometric parameters and psoas major muscle thickness in the controls was reported; and a comparison of the thickness of the psoas major muscle between the control group and artisan (quasi-experimental) group was also reported. The Sonographs showing the PMMT in the respective groups are presented in Figures 2 and 3.

Table I shows the 95\% confidence interval of psoas major muscle thickness $(\mathrm{mm})$ in the study population. The psoas major muscle thickness range is $21.0-48.8 \mathrm{~mm}$, with mean $31.7 \mathrm{~mm}$ and standard deviation of $7.9 \mathrm{~mm}$ and the $95 \%$ confidence interval of $28.0 \mathrm{~mm}-35.4 \mathrm{~mm}$.

Table II shows the relationship between thickness of the psoas major muscle $(\mathrm{mm})$ and other anthropometric parameters of the control group. There is no correlation between age and thickness of psoas major $(r=0.059, p>0.05)$. Also there is no correlation between height and thickness of psoas major muscle $(r=-0.18, p>0.05)$, nor between weight and thickness of psoas major muscle $(r=$ $-0.244, p>0.05$ ). In the same vein, there is no significant correlation between body mass index (BMI) and thickness of psoas major muscle $(r=-0.151$, 


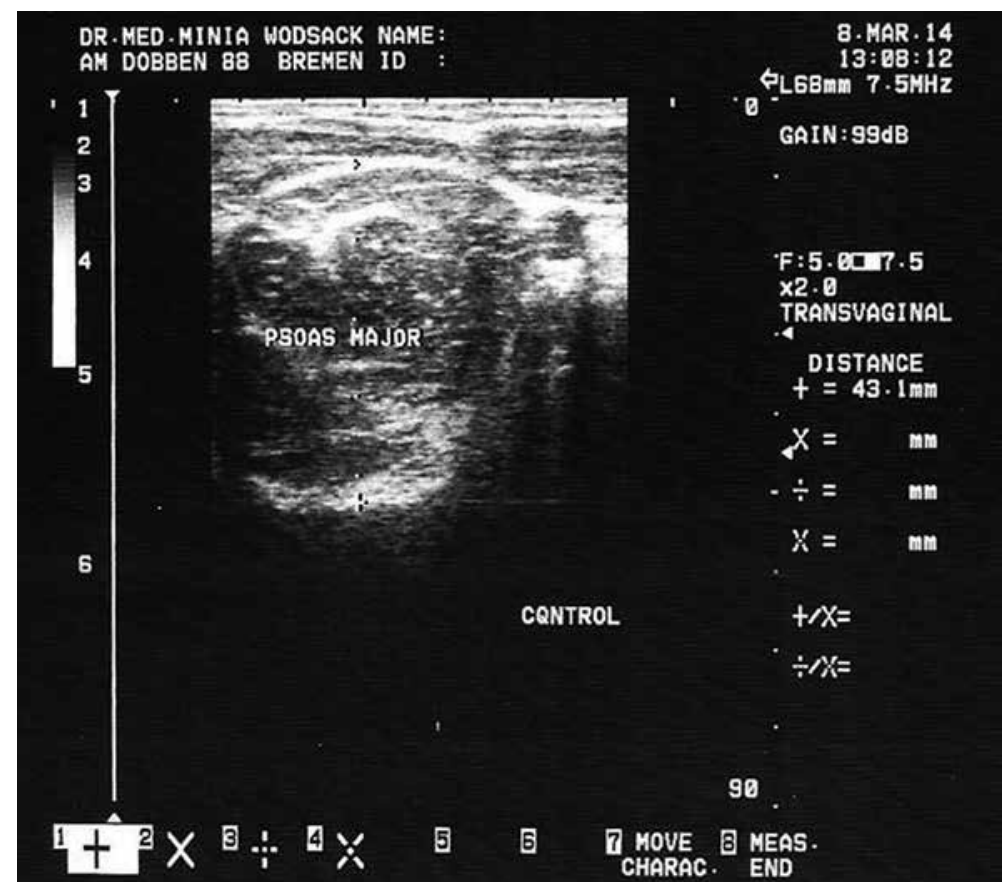

Figure 2. Sonograph of control group

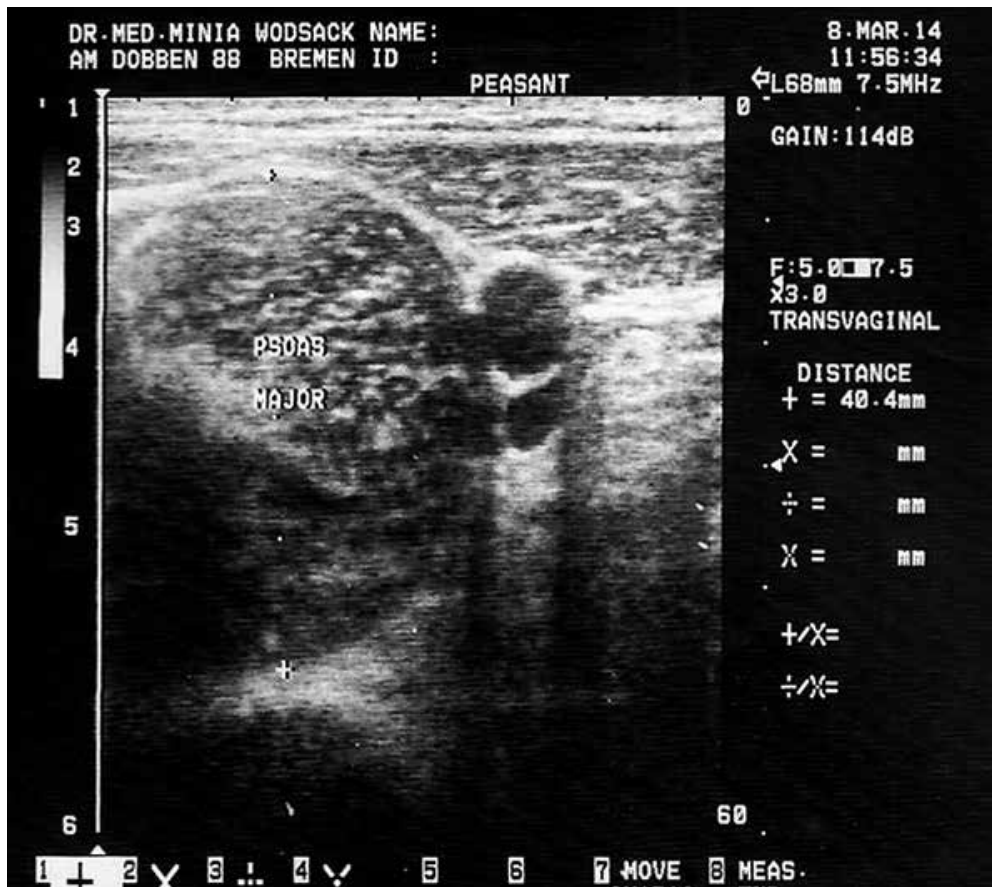

Figure 3. Sonograph of artisan (labourers) group

Table I. Determination of thickness of psoas major muscle thickness in control group

\begin{tabular}{|c|c|c|c|c|c|c|}
\hline \multirow[t]{2}{*}{ Parameters } & \multirow[t]{2}{*}{ Minimum } & \multirow[t]{2}{*}{ Maximum } & \multirow[t]{2}{*}{ Mean } & \multirow{2}{*}{$\begin{array}{l}\text { Standard } \\
\text { deviation }\end{array}$} & \multicolumn{2}{|c|}{ 95\% confidence interval } \\
\hline & & & & & Lower value & Upper value \\
\hline $\begin{array}{l}\text { Psoas major muscle } \\
\text { thickness }[\mathrm{mm}]\end{array}$ & 21.0 & 48.8 & 31.735 & 7.9145 & 28.031 & 35.439 \\
\hline
\end{tabular}

$p>0.05)$; and there is no significant correlation between body surface area (BSA) and thickness of psoas major muscle $(r=-0.255, p>0.605)$.
Table III shows the comparison of psoas major muscle thickness between control group and artisan group. The mean and standard deviation 
Table II. Relationship between thickness of psoas major muscle $(\mathrm{mm})$ and anthropometric parameters of the control group

\begin{tabular}{|lcc|}
\hline Anthropometric parameters & \multicolumn{2}{c|}{ Thickness of psoas major muscle $[\mathrm{mm}]$} \\
\cline { 2 - 3 } & Correlation coefficient $(r)$ & $P$-value \\
\hline Age [years] & 0.159 & 0.502 \\
\hline Height $[\mathrm{m}]$ & -0.186 & 0.432 \\
\hline Weight $[\mathrm{kg}]$ & -0.6244 & 0.299 \\
\hline Body mass index $[\mathrm{kg} / \mathrm{m}]$ & -0.151 & 0.525 \\
\hline Body surface area & -0.255 & 0.278 \\
\hline
\end{tabular}

Table III. Comparison between thickness of psoas major muscle of control group and artisan group using student's t-test

\begin{tabular}{|c|c|c|c|c|c|}
\hline Parameters & Control, mean \pm SD & Artisan, mean \pm SD & $T$-test & $\mathrm{D} f$ & $P$-value \\
\hline $\begin{array}{l}\text { Psoas major muscle } \\
\text { thickness }[\mathrm{mm}]\end{array}$ & $31.74 \pm 7.91$ & $40.10 \pm 4.89$ & -4.020 & 38 & $<0.001$ \\
\hline
\end{tabular}

of thickness of psoas major muscle in control and peasant are $31.74 \pm 7.91 \mathrm{~mm}$ and $40.10 \pm 89 \mathrm{~mm}$ respectively and there is a significant difference between them $(p<0.05)$.

\section{Discussion}

The ultrasonographic measurement of psoas major muscle thickness was determined among young male adults in a south-eastern Nigerian population. The normal thickness of psoas major muscles in the control group (people who do not engage in hard labour) was established. Also the thickness of the muscle among the artisan group (motor mechanics and masons who engage in hard labour such as lifting heavy motor engines, big hammers, building blocks, concrete filled head pans and other hard labour) was determined and comparisons made between them.

The results of the present study reveal that the normal thickness (nomogram) of the psoas major muscle in the control group is $31.7 \pm 7.91 \mathrm{~mm}$ (range: $21.0-48.8 \mathrm{~mm}$ ). The knowledge of the nomogram of the psoas major muscle could therefore be applied by physiotherapists and musculoskeletal therapists as a biofeedback tool in functional retraining of the psoas major in sports and occupational medicine. Therefore, timely assessment of the muscle nomogram using ultrasound is particularly important for timely detection of a contracted (or shortened) psoas major which leads to lower back pain and overuse injuries among the general population [8]. The findings from this present study could serve as a functional guide for further understanding of muscle morphology and its dynamism in force generation and possible role during environmental, occupational and sportsbased biomechanical interactions.
The relationship of psoas major muscle thickness with other anthropometric parameters was investigated in the present study. The findings revealed no correlation between age and thickness of the psoas major muscle $(r=0.159, p>0.05)$. This means that age had no significant effect on the thickness of the psoas major muscle as revealed in this study. This could be a result of the narrow age range used for this study. Also weight as an anthropometric parameter had no significant correlation with the thickness of the psoas major muscle $(r=-0.244, p>0.05)$. Also there was no significant correlation between height and thickness of the psoas major muscle $(r=-0.186$, $p>0.05$ ).

In the same vein, there was no significant correlation between $\mathrm{BMI}$ and thickness of the psoas major muscle ( $r=-0.151, p>0.05)$, and there was also no significant correlation between BSA and thickness of the psoas major muscle $(r=-0.255$, $p>0.05)$. These non-relationships may be an indication that the architectural disposition of the psoas major may not be a factor in the overall stature of individuals but may rather be an important factor in the postural inclination of the individual.

The results of the present study reveal that the normal thickness (nomogram) of the psoas major muscle in the control group is $31.7 \pm 7.91 \mathrm{~mm}$ (range: $21.0-48.8 \mathrm{~mm}$ ), while that of the artisans in the present study is $40.10 \pm 4.89 \mathrm{~mm}$ (range: 30.0-50.7 mm). This shows a significant difference in the psoas major muscle thickness between the groups $(p<0.05)$. The difference in the thickness of the psoas major muscle between the control group (relatively sedentary students who do not engage in manual or hard labour) and the artisan group (quasi-experimental group - labourers who engage in manual or hard labour 
jobs) could be a result of lifting of heavy objects such as heavy motor engines, heavy hammers and numerous unregulated exercises carried out by the subjects in this quasi-experimental group. Others may include lifting building blocks, pulling or pushing heavy weight materials, sit-up exercises during laying bricks and other related activities associated with the type of work they engage in.

Muscle thickness is likely to be affected by the manner in which force is transmitted, and the rate of change in muscle thickness is dependent on the muscle in question [17]. And since the psoas major is important during the act of lifting because of its pivotal role in trunk stability, this assertion can be applied to the muscle under study. However, the psoas major muscle is likely to be mainly affected by the manner in which force is transmitted during pushing, pulling or sit-up exercises. Although change in human muscle thickness is not always predictable but instead appears to be muscle specific [18], the findings from the present study are specific for the psoas muscle and are in conformity with a previous report [19] as regards muscle thickness.

From the above, it is therefore possible that this continuous stretching and overuse of the muscle in lumbopelvic mechanics associated with hard labour experienced by the artisan group could have caused the increased thickness seen in the psoas major muscle. A long-term assessment of this occupation-related thickening could distort the alignment of associated musculoskeletal components of the posterior abdominal wall and could be a risk factor in the future occurrence of low back pain. This is also supported by the fact that a sedentary life style (prolonged sitting down) contracts the psoas major muscle, which is seen in the control group, having a lower psoas muscle thickness as compared to the artisan group.

In conclusion, the study, as a novelty, has shown that unregulated occupational related physical activity among artisans (hard labourers) increases the thickness of the psoas major muscle in comparison to a relatively sedentary age-matched student group. This is a conclusive indication that this unregulated physical activity increases psoas muscle thickness and the increase could trigger distortions in the normal lumbopelvic mechanics of the affected individuals.

\section{Conflict of interest}

The authors declare no conflict of interest.

\section{References}

1. Juker D, McGill SM, Kropf P, Steffen T. Quantitative intramuscular myoelectric activity of lumbar portions of pso- as and abdominal wall during a wide variety of tasks. J Med Sci Sports 1998; 30: 301-10.

2. Moseley GL, Hodges PW, Gandevia SC. Deep and superficial fibers of the lumbar multifidus muscle are differentially active during voluntary arm movements. Spine 2002; 27: e29-36.

3. Little TL, Mamser J. Low back pain associated with internal snapping hip syndrome in competitive cyclist. $\mathrm{Br}$ J Sports Med 2008; 42: 308-9.

4. Aagard P, Andersen JL, Dyhre-Poulsen P, et al. A mechanism foe increased contractile strength of human pinnate muscle in response to strength training: changes in muscles architecture. J Physiol 2001; 53: 613-23.

5. Quint U, Wilke HJ, Shirazi AA, Parniapour M, Loer F, Claes LE. Importance of the stability of the lumbar spine. Spine 1998; 23: 1937-45.

6. Johston CAM, Wiley JP, Lindsay DM, Wiseman DA. Iliopsoas bursitis and tendinitis: a review. Sports Med 1998; 25: 271-83.

7. LaBan MM. Iliopsoas weakness: a clinical sign of lumbar spinal stenosis. Am J Physiol Med Rehabil 2004; 83: 224-5.

8. Wild JJ, Neal D. Use of high frequency ultrasonic waves for detecting changes of texture in living tissue. Lancet 1957; 1: 655-7.

9. Fornage BD. The cause for ultrasound of muscles and tendons. Semin Musculoskelet Radiol 2000; 4: 375-91.

10. Bogduk N, Pearcy M, Hadfield G. Anatomy and biomechanics of psoas major. J Clin Biomech 1992; 7: 109-19.

11. Egwu OA, Njoku CO, Ewunonu EO, Ukoha UU, Eteudo $\mathrm{N}$, Mgbechi CE. Assessment of massteter muscle thickness in adult nigeraian population: an ultrasound based study. Int J Biomed Res 2012; 3: 143-6.

12. Nourbakhsh MR, Arab AM. Relationship between mechanical factors and incidence of low back pain. J Orthoped Sports Phys Therapy 2002; 32: 447-60.

13. Richardson C, Jull G, Hodges P, Hides J. Therapeutic exercise for spinal segmental stabilization in low back pain: scientific maneuvers. J Appl Physiol 1997; 83: 753-60.

14. Petrous P. Ultrasound of muscles. Eur J Radiol 2002; 12 : 35-43.

15. Egwu OA, Bello E, Njoku CO. In vivo assessment of the diaphragm in young male healthy adults: occupationbased activity-related differences. Studia Medyczne 2014; 30: 221-7.

16. Sofia B, Anna N, Marita H, Christer S, Yiva A. Ultrasound evaluation in combination with figer force measurements of the forearm musculus extensor digitorum communis in healthy subjects. BMC Med Imaging 2008; 8: 1-4.

17. Maganaris CN, Baltzopoulos V, Sarageant AJ. In vivo measurement of tricips surea complex architecture in man: implication of muscles fuction. J Physiol 1998; 2: 603-14.

18. Sigrid P. Skeletal muscle ultrasound. Eur Translat J Myology 2010; 1: 145-55.

19. Rutherford MO, Jones DA. Measurement of fiber pennation using ultrasound in the human quadriceps in vivo. Eur J Appl Physiol 1992; 65: 433-7. 